\title{
Neoadjuvant chemotherapy-induced decrease of prognostic nutrition index predicts poor prognosis in patients with breast cancer
}

Takaaki Oba, Kazuma Maeno ${ }^{*}$, Daiya Takekoshi, Mayu Ono, Tokiko Ito, Toshiharu Kanai and Ken-ichi Ito

\begin{abstract}
Background: The prognostic nutritional index (PNI), which is an easily calculated nutritional index, is significantly associated with patient outcomes in various solid malignancies. This study aimed to evaluate the prognostic impact of PNI changes in patients with breast cancer undergoing neoadjuvant chemotherapy (NAC).

Methods: We reviewed patients with breast cancer who underwent NAC and a subsequent surgery for breast cancer between 2005 and 2016. PNI before and after NAC were calculated using the following formula: $10 \times$ serum albumin $(\mathrm{g} / \mathrm{dl})+0.005 \times$ total lymphocyte count $/ \mathrm{mm}^{3}$. The relationship between $\mathrm{PNI}$ and prognosis was retrospectively analyzed.

Results: In total, 191 patients were evaluated. There was no significant difference in disease-free survival (DFS) between the pre-NAC PNI high group and the pre-NAC PNI low group (cutoff: 53.1). However, PNI decreased in 181 patients (94.7\%) after NAC and the mean PNI also significantly decreased after NAC from $52.6 \pm 3.8$ pre-NAC to $46.5 \pm 4.4$ post-NAC $(p<0.01)$. The mean $\triangle \mathrm{PNI}$, which was calculated as pre-NAC PNI minus post-NAC PNI, was 5.4. The high $\triangle \mathrm{PNI}$ group showed significantly poorer DFS than the low $\triangle \mathrm{PNI}$ group (cut off: 5.26$)(p=0.015)$. Moreover, high $\triangle \mathrm{PNI}$ was an independent risk factor of DFS on multivariate analysis $(p=0.042)$.

Conclusions: High decrease of PNI during NAC predicts poor prognosis. Thus, maintaining the nutritional status during NAC may result in better treatment outcomes in patients with breast cancer.
\end{abstract}

Keywords: Prognostic nutritional index, Disease-free survival, Neoadjuvant chemotherapy, Breast cancer

\section{Background}

Despite recent improvements in early detection and progress in surgical techniques, chemotherapy, molecular targeting therapy, and endocrine therapy, breast cancer remains the leading cause of cancer death for women [1]. That is why some patients with breast cancer still develop recurrence even after curative resection and neoadjuvant/adjuvant therapy. Therefore, prevention of recurrence and accurate prediction of prognosis are needed to improve patient survival and fully inform patients.

\footnotetext{
* Correspondence: kmaeno@shinshu-u.ac.jp

Division of Breast and Endocrine Surgery, Department of Surgery, Shinshu University School of Medicine, 3-1-1 Asahi, Matsumoto, Nagano, Japan
}

Accumulating evidence suggests that nutritional status has a strong impact on the outcome of cancer treatment [2]. The prognostic nutritional index (PNI), which is calculated via a simple formula using only serum albumin level and lymphocyte cell count in the peripheral blood, is among the most commonly used parameters to evaluate nutritional status [3]. It has been demonstrated that a preoperative low PNI status is both a risk factor for postoperative complications and a predictive factor for poor prognosis among patients with various malignant tumors including gastric, colorectal, lung, pancreatic, and renal cell cancer undergoing surgery [4-10]. However, only few nutritional studies in the treatment for breast cancer have been conducted [11, 12]. Therefore, the significance of PNI in breast cancer still remains unclear. 
Neoadjuvant chemotherapy (NAC) has become widely used for patients with locally advanced breast cancer because it has been shown to significantly elevate the rate of breast-conserving surgery by reducing the tumor volume. Further, the prognosis of the patients who underwent NAC is not inferior to those treated with postoperative chemotherapy [13]. In addition to these clinical benefits, NAC also provides important prognostic information such as pathological complete response (pCR) rate, which has been demonstrated to be a prognostic marker in human epidermal growth factor receptor type 2 (HER2)-positive or triple-negative breast cancer (TNBC) [14]. In this regard, NAC could have potential to present other various prognostic markers as well as pCR and we focused on PNI.

It has been reported that chemotherapy leads to malnutrition due to its gastrointestinal adverse effects including anorexia, nausea, vomiting, stomatitis, and diarrhea [15]. Migita et al. reported that a decrease of PNI during NAC in patients with gastric cancer is associated with a worse long-term outcome [16]. However, to date, there has been no study on the impact of changes in PNI on postoperative prognosis in patients with breast cancer who underwent NAC.

As such, the present study aimed to evaluate the prognostic impact of PNI and other nutritional indices in patients with breast cancer. Towards this goal, we evaluated the changes in PNI and other nutritional factors (e.g., serum albumin level and neutrophil/lymphocyte ratio (NLR)) and body mass index (BMI) during NAC and investigated the association between them and patient outcomes.

\section{Methods}

\section{Patients and study design}

This retrospective, single-center study evaluated patients with breast cancer who underwent NAC and subsequent surgery in Shinshu University Hospital between 2005 and 2016. Patients who could not provide detailed laboratory data and those who could not complete NAC or required a treatment delay of $\geq 2$ weeks due to chemotoxicity were excluded.

\section{Data collection}

Data on clinicopathological characteristics, including age, sex, clinical stage at diagnosis, histological type, histological grade (HG), estrogen receptor (ER), progesterone receptor (PgR), HER2 status, NAC regimens, operation procedure, pathological responses to NAC, and presence of recurrence, were collected from the patients' medical records. Disease-free survival was defined as the time from surgery to the date of locoregional relapse or distant metastases, whichever occurred first.
PNI, the serum albumin level (Alb) (g/dl), NLR, and BMI were used as nutritional parameters in this study. Pre- and post-NAC blood examination data were also obtained. In addition, both body weight and height were obtained at the same day when blood samples were collected. Pre-NAC nutritional values were collected more than 1 week before the beginning of NAC, while postNAC values were collected at more than 4 weeks after the last administration of NAC. PNI values were calculated using the following formula: $10 \times$ serum albumin value $(\mathrm{g} / \mathrm{dl})+0.005 \times$ total lymphocyte counts in the peripheral blood $/ \mathrm{mm}^{3}$ [3]. NLR values were as the total neutrophil count divided by the total lymphocyte counts, while BMI as patient's weight (in kilograms) divided by the square of height (meters) $[17,18] . \Delta \mathrm{PNI}, \Delta \mathrm{Alb}$, $\triangle \mathrm{NLR}$, and $\triangle \mathrm{BMI}$ were calculated as each value on preNAC minus that on post-NAC. The receiver operating characteristic (ROC) curve of each prognostic parameter was analyzed to determine the best cut-off value for disease-free survival.

\section{NAC regimens and surgical methods}

Two different NAC regimens were mainly used: (1) anthracycline-based regimens (AC) including EC (60-75 $\mathrm{mg} / \mathrm{m}^{2}$ epirubicin and $600 \mathrm{mg} / \mathrm{m}^{2}$ cyclophosphamide) or FEC $\left(500 \mathrm{mg} / \mathrm{m}^{2}\right.$ fluorouracil, $75-100 \mathrm{mg} / \mathrm{m}^{2}$ epirubicin, and $500 \mathrm{mg} / \mathrm{m}^{2}$ cyclophosphamide) administered every 3 weeks and (2) taxane regimens including triweekly administered docetaxel (DOC) $75 \mathrm{mg} / \mathrm{m}^{2}$ or weekly administered paclitaxel (PTX) $80 \mathrm{mg} / \mathrm{m}^{2}$. Most of the patients who underwent four cycles of $\mathrm{AC}$ were then administered a further four cycles of DOC or PTX. In HER2positive patients who received taxane regimens, $6 \mathrm{mg} / \mathrm{kg}$ (triweekly) or $2 \mathrm{mg} / \mathrm{kg}$ (weekly) trastuzumab was simultaneously administered. Surgery was performed within 4-7 weeks after NAC completion. All patients underwent axillary lymph node dissection. The efficacy of NAC was pathologically examined in the surgical specimens. pCR was defined as no evidence of residual invasive carcinoma in the breast tissue regardless of the axillary lymph node status.

\section{Adjuvant trastuzumab, endocrine, and radiation therapy after surgery}

Following surgery, extensional adjuvant trastuzumab (initially $8 \mathrm{mg} / \mathrm{kg}$, followed by $6 \mathrm{mg} / \mathrm{kg}$ ) was administered every 3 weeks for 12 months to patients with HER2-positive breast cancer. Whole breast irradiation of 50-60 Gy was performed for the patients who underwent breastconserving surgery, while chest wall and regional lymph node irradiation of 50-60 Gy was performed for the patients with more than three nodal metastases on the postoperative pathological examinations or preoperative imaging examinations including ultrasonography, magnetic 
resonance imaging, and ${ }^{18}$ F-fluorodeoxyglucose positron emission tomography. In addition, postmenopausal patients with positive ER or PgR status were treated with aromatase inhibitors for more than 5 years, whereas premenopausal patients were treated with tamoxifen or tamoxifen with luteinizing hormone-releasing hormone agonist.

\section{Statistical analysis}

Categorical and continuous variables were analyzed using Fisher's exact test and two-sided tests, respectively. Survival curves were estimated using the Kaplan-Meier method, and significant differences in survival were assessed using the log-rank test. Univariate and multivariate analyses with a Cox proportional hazards model were performed to determine significant factors. All statistical analyses were carried out using StatFlex ver.6 (Artech Co., Ltd., Osaka, Japan), and $p<0.05$ was considered statistically significant.

\section{Results}

\section{Clinicopathological characteristics and nutrition} parameter of patients

In total, 191 patients with a mean age ( \pm standard deviation) of $51.2 \pm 10.4$ were evaluated. The patient characteristics are shown in Table 1 . With respect to clinical stage at diagnosis, 1 (0.5\%), 118 (61.8\%), and $72(37.7 \%)$ patients had stage I, II, and III disease, respectively. For the pathological classification, 171 patients $(89.5 \%)$ had invasive ductal carcinoma; 12 patients (6.3\%), invasive lobular carcinoma; and 8 patients (4.2\%), other special types. As for intrinsic subtype, 107 cases (56.0\%) were luminal

Table 1 Clinicopathologic characteristics in patients

\begin{tabular}{|c|c|c|c|c|c|}
\hline \multicolumn{2}{|l|}{ Variables } & $\begin{array}{l}\text { Total } \\
n=191(\%)\end{array}$ & $\begin{array}{l}\text { low } \Delta \text { PNI } \\
n=91(\%)\end{array}$ & $\begin{array}{l}\text { high } \Delta \mathrm{PNI} \\
n=100(\%)\end{array}$ & $p$ value \\
\hline Age $($ mean $\pm S D)$ & & $51.2 \pm 10.4$ & $50.9 \pm 9.26$ & $51.5 \pm 11.3$ & 0.68 \\
\hline Sex(Male/Female) & & 0/191 & 0/91 & 0/100 & \\
\hline \multirow[t]{4}{*}{ Pre-NAC clinical stage } & I & $1(0.5 \%)$ & $1(1.1 \%)$ & $0(0 \%)$ & 0.67 \\
\hline & $\|$ & $118(61.8 \%)$ & $52(57.2 \%)$ & $66(66.0 \%)$ & \\
\hline & III & $72(37.7 \%)$ & $38(41.7 \%)$ & $34(34.0 \%)$ & \\
\hline & IV & $0(0 \%)$ & $0(0 \%)$ & $0(0 \%)$ & \\
\hline \multirow[t]{3}{*}{ Histological type } & IDC & $171(89.5 \%)$ & 81 (89.0\%) & $90(90.0 \%)$ & 0.89 \\
\hline & ILC & $12(6.3 \%)$ & 7 (7.7\%) & $5(5.0 \%)$ & \\
\hline & Special type & $8(4.2 \%)$ & $3(3.3 \%)$ & $5(5.0 \%)$ & \\
\hline \multirow[t]{4}{*}{$\mathrm{HG}$} & 1 & $54(28.3 \%)$ & $18(19.9 \%)$ & $36(36.0 \%)$ & 0.15 \\
\hline & 2 & 89 (46.6\%) & $47(51.6 \%)$ & $42(42.0 \%)$ & \\
\hline & 3 & $23(12.0 \%)$ & $14(15.3 \%)$ & $9(9.0 \%)$ & \\
\hline & Unknown & $25(13.1 \%)$ & $12(13.2 \%)$ & $13(13.0 \%)$ & \\
\hline \multirow[t]{4}{*}{ Subtype } & Lunimal & 107 (56.0\%) & $51(56.0 \%)$ & $56(56.0 \%)$ & 0.79 \\
\hline & Luninal HER2 & 37 (19.4\%) & 19 (20.9\%) & $18(18.0 \%)$ & \\
\hline & HER2 enriched & $24(12.6 \%)$ & $12(13.2 \%)$ & $12(12.0 \%)$ & \\
\hline & TNBC & $23(12.0 \%)$ & $9(9.9 \%)$ & $14(14.0 \%)$ & \\
\hline \multirow[t]{3}{*}{ Regimens of NAC } & $A C$ & $11(5.8 \%)$ & $10(11.0 \%)$ & $1(1.0 \%)$ & 0.02 \\
\hline & $\mathrm{AC} \rightarrow \mathrm{PTX}$ and/or HER & $91(47.6 \%)$ & $52(57.2 \%)$ & 39 (39.0\%) & \\
\hline & $\mathrm{AC} \rightarrow \mathrm{DOC}$ and/or HER & 89 (46.6\%) & $29(31.8 \%)$ & $60(60.0 \%)$ & \\
\hline \multirow[t]{2}{*}{ Operation procedures } & $\mathrm{Bt}, \mathrm{Ax}$ & $128(67.1 \%)$ & $59(64.8 \%)$ & 69 (69.0\%) & 0.65 \\
\hline & $\mathrm{Bp}, \mathrm{Ax}$ & $63(32.9 \%)$ & $32(35.2 \%)$ & $31(31.0 \%)$ & \\
\hline \multirow[t]{2}{*}{ Pathological response to NAC } & non-pCR & $154(80.6 \%)$ & $78(85.7 \%)$ & $76(76.0 \%)$ & 0.10 \\
\hline & $\mathrm{pCR}$ & $37(19.4 \%)$ & $13(14.3 \%)$ & $24(24.0 \%)$ & \\
\hline Pre-NAC PNI & & $52.6 \pm 3.8$ & $50.9 \pm 3.7$ & $54.1 \pm 3.6$ & $<0.01$ \\
\hline Post-NAC PNI & & $46.5 \pm 4.5$ & $48.8 \pm 3.9$ & $44.5 \pm 4.0$ & $<0.01$ \\
\hline Recurrence & & 38 (19.9\%) & $13(14.3 \%)$ & $25(25.0 \%)$ & 0.06 \\
\hline
\end{tabular}

NAC Neoadjuvant chemotherapy, HG Histological grade, IDC Invasive ductal carcinoma, ILC Invasive lobular carcinoma, AC Antracycline, PTX paclitaxel, DOC docetaxel, HER Trastuzumab, Bt Mastectomy, Bp Partial resection of breast, Ax Axillary dissection, NAC neoadjuvant chemotherapy, PNI prognostic nutritional index 


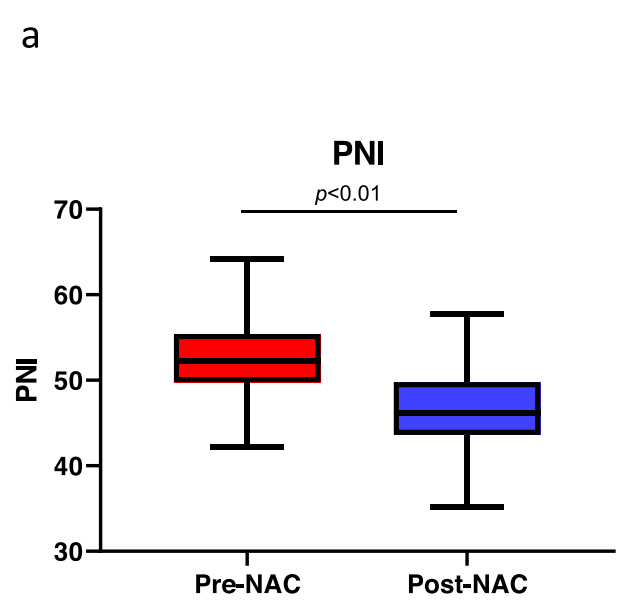

b

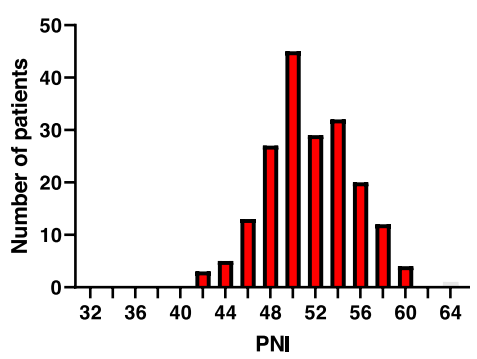

C

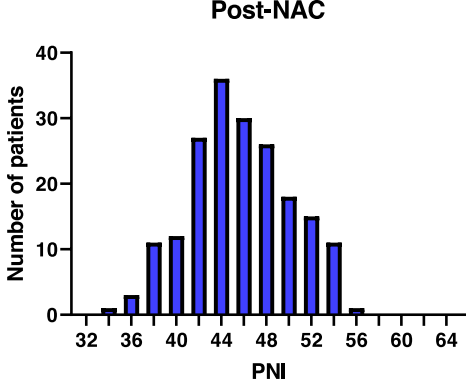

Fig. 1 Box-and-whisker plot for Pre-NAC and post-NAC PNI ( $p<0.01)$ (a). Distribution of pre-NAC (b) and post-NAC PNI (c). NAC: Neoadjuvant chemotherapy, PNI: Prognostic nutritional index

(ER+ and/or PgR+/HER2-), 37 cases (19.4\%) were luminal HER-2 (ER+ and/or PgR+/HER2+), 24 cases (12.6\%) were HER2 enriched (ER- and PgR- / HER2+), and 23 cases (12.0\%) were TNBC (ER- and PgR-/HER2-). Eleven patients (5.8\%) were treated with AC without taxane; 91 patients (47.6\%), AC followed by weekly PTX and/or trastuzumab; and 89 patients (46.6\%), AC followed by triweekly DOC and/or trastuzumab. Regarding chemotoxicity, 14 patients $(7.3 \%)$ required a dose reduction of $<20 \%$ during NAC. Mastectomy was performed for 128 patients (67.1\%), while breast-conserving surgery was performed for 63 patients (32.9\%). pCR was obtained in 37 patients (19.4\%). The median follow-up period after surgery was 51 months (range, $1-151$ months), and 38 patients (19.9\%) developed recurrence.

The mean PNI (pre: $52.6 \pm 3.8$ vs post: $46.5 \pm 4.5$; $p<$ 0.01 ) and Alb (pre: $4.41 \pm 0.30$ vs post: $4.11 \pm 0.36$; $p<$ 0.01 ) were significantly decreased after NAC, whereas NLR was significantly increased after NAC (pre: $2.50 \pm$ 1.4 vs post: $2.96 \pm 1.6 ; p<0.01$ ). Meanwhile, there was no significant difference in BMI before and after NAC (pre: $22.5 \pm 3.9$ vs post: $22.3 \pm 3.9 ; p=0.63$ ) (Fig. 1 , Additional file 1: Figure S1, Table 2). Among these four factors, PNI was the most commonly decreased (181/191; 94.7\%) (Additional file 2: Table S1).

\section{Association between nutritional parameters and disease- free survival}

Disease-free survival in the high and low groups of each nutritional parameter was analyzed to examine the correlation between nutritional status and patient outcome. The optimal cutoff values of PNI, Alb, NLR, and BMI for disease-free survival as identified using the ROC curves were 53.1, 4.36, 2.32, and 21.7 for pre-NAC, respectively, and 45.4, 4.04, 2.57 and 21.5, respectively, for post-NAC (Additional file 3: TableS2). In pre-NAC, there were no significant differences in disease-free survival between the high and low groups for each nutritional parameter $(p=0.89$ for PNI, $p=0.65$ for Alb, $p=$ 0.25 for NLR, and $p=0.76$ for BMI) (Fig. 2a, Additional file 4: Figure S2). Similar findings were found on post-NAC ( $p=0.21$ for PNI, $p=0.78$ for Alb, $p=0.58$ for NLR, and $p=0.58$ for BMI) (Fig. 2b, Additional file 5: Figure S3). As well as disease-free survival, diseasespecific survival was not different between the high and low groups for each nutritional parameter (Pre-NAC: $p=0.21$ for PNI, $p=0.65$ for Alb, $p=0.068$ for NLR, and $p=0.43$ for BMI, Post-NAC: $p=0.98$ for PNI, $p=0.14$ for Alb, $p=0.57$ for NLR, and $p=0.80$ for BMI) (Additional file 6: Figure S4).

Table 2 Comparison of nutritional factors before and after NAC (mean \pm standard deviation)

\begin{tabular}{llll}
\hline Variables & Pre-NAC & Post-NAC & $p$ value \\
\hline PNI & $52.6 \pm 3.8$ & $46.5 \pm 4.5$ & $<0.01$ \\
Serum albumin level (g/dl) & $4.41 \pm 0.30$ & $4.11 \pm 0.36$ & $<0.01$ \\
NLR & $2.50 \pm 1.4$ & $2.96 \pm 1.6$ & $<0.01$ \\
BMI & $22.5 \pm 3.9$ & $22.3 \pm 3.9$ & 0.63 \\
\hline
\end{tabular}

NAC Neoadjuvant chemotherapy, PNI Prognostic nutritional index, NLR Neutrophil/lymphocyte ratio, BMI Body mass index 
a

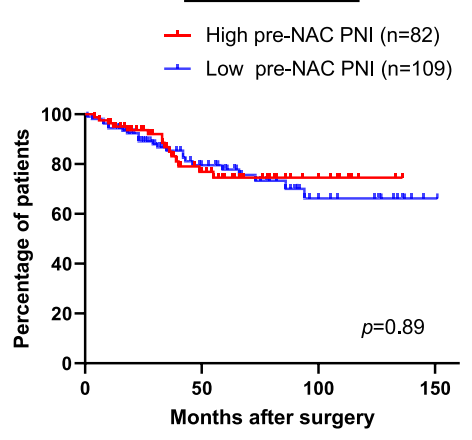

HR:0.91 (95\%Cl:0.47-1.72) b

Post-NAC PNI

- High post-NAC PNI ( $\mathrm{n}=108)$

- Low post-NAC PNI $(n=83)$

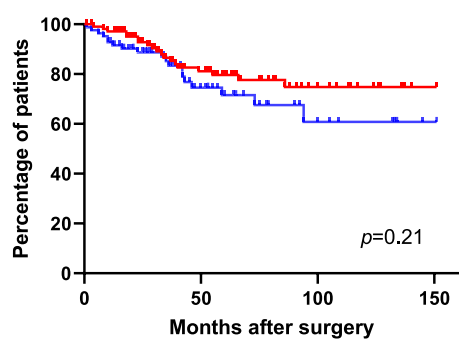

HR:0.67 (95\%Cl:0.35-1.28)

Fig. 2 Kaplan-Meier curves for DFS according to PNI at (a) pre- $(p=0.89)$ and (b) post-NAC $(p=0.21)$. DFS: Disease-free survival, NAC: Neoadjuvant chemotherapy, PNI: Prognostic nutritional index

\section{Association between changes of nutrition parameters during NAC and disease-free survival}

Next, we focused on the association between changes in nutrition parameters during NAC and disease-free survival. The optimal cutoff value determined via the ROC curve for disease-free survival was 5.26 for $\triangle \mathrm{PNI}, 0.34$ for $\triangle \mathrm{Alb},-0.17$ for $\triangle \mathrm{NLR}$, and -0.26 for $\triangle \mathrm{BMI}$ (Additional file 3: TableS2). Interestingly, the high $\triangle \mathrm{PNI}$ group had significantly poorer disease-free survival than the low $\Delta$ PNI group $(p=0.015)$ (Fig. 3). Additionally, a

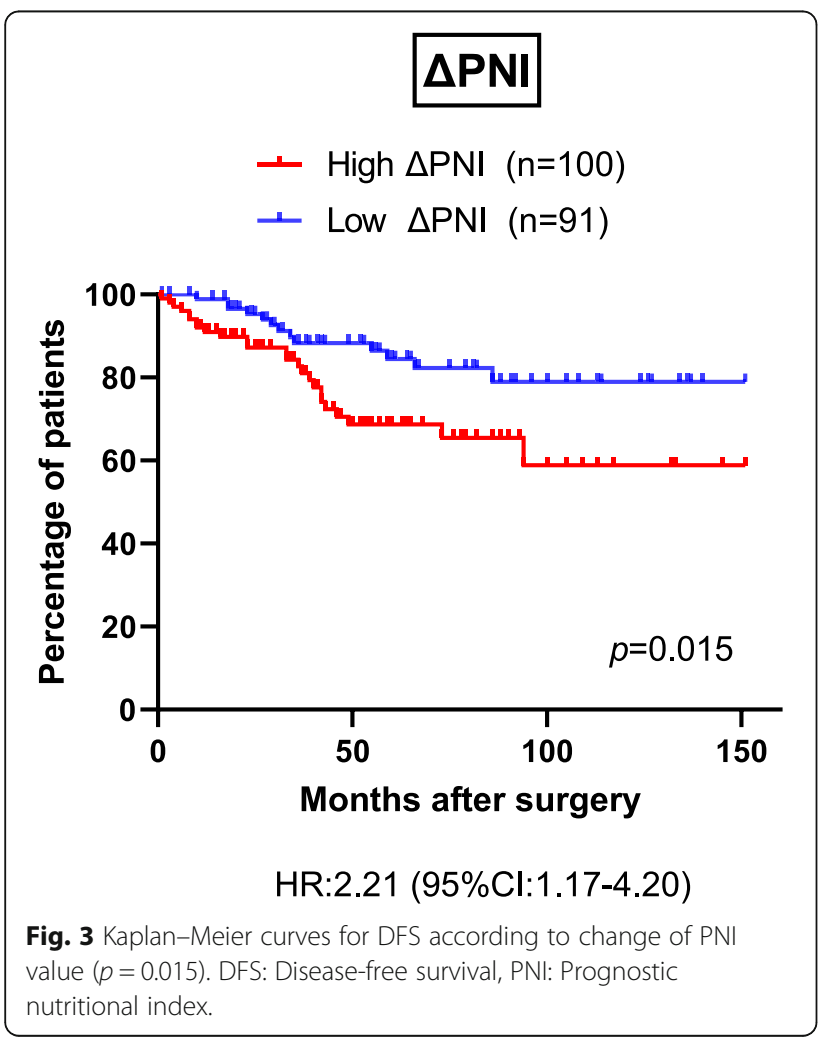

trend for lower disease-specific survival was found in the high $\triangle \mathrm{PNI}$ group than in the low $\triangle \mathrm{PNI}$ group, although no statistical difference was observed $(p=0.14)$ (Additional file 7: Figure S5). Meanwhile, there were no significant differences in either disease-free survival or disease-specific survival between the high and low groups according to $\Delta \mathrm{Alb}(p=0.053$ for disease-free survival, $p=0.14$ for disease-specific survival), $\Delta$ NLR $(p=$ 0.65 for disease-free survival, $p=0.20$ for disease-specific survival), and $\triangle \mathrm{BMI}(p=0.66$ for disease-free survival, $p=0.66$ for disease-specific survival) (Additional file 8: Figure S6, Additional file 9: Figure S7).

The clinicopathological characteristics of the high and low $\triangle$ PNI groups are shown in Table 1 . The median follow-up period after surgery was 64 (3-151) months for the high $\triangle \mathrm{PNI}$ group and $43(1-151)$ for the low $\triangle \mathrm{PNI}$ group. The mean age, clinical stage, histological type, HG, subtype, operation procedure, and pathological response to NAC were not significantly different between the two groups. Meanwhile, NAC regimens differed significantly, with a higher rate of patients who underwent NAC with DOC in the high $\triangle \mathrm{PNI}$ group $(p=0.02)$. Recurrence was more frequent in the high $\triangle \mathrm{PNI}$ group with marginal significance $(p=0.06)$. In the high $\triangle \mathrm{PNI}$ group, pre-NAC PNI was significantly higher than that in the low $\Delta$ PNI group $(p<0.01)$. Furthermore, the mean $\triangle \mathrm{PNI}$ was significantly higher in the patients with high pre-NAC PNI than in those with low preNAC PNI (Additional file 10: Figure S8), indicating that a large PNI change may be likely to occur in patients with high PNI at baseline.

The higher proportion of patients treated with DOC in the high $\triangle \mathrm{PNI}$ group prompted us to examine whether NAC regimens affected disease-free survival. However, we found no significant difference in diseasefree survival among the three NAC regimens (AC, AC followed by PTX and/or trastuzumab, or AC followed by 
DOC and/or trastuzumab) (Additional file 11: Figure S9). These data suggest that the difference in diseasefree survival by $\triangle \mathrm{PNI}$ is independent of NAC regimens.

Next, we examined if tumor burden at time of the diagnosis could influence the pre-NAC PNI, post-NAC PNI, or $\triangle$ PNI. When we divided the patients into stage I, II and stage III, pre-NAC PNI, post-NAC PNI, or $\Delta$ PNI were not different $(p=0.87, p=0.73$, and $p=0.85$, respectively), indicating that the volume of disease did not affect either the PNI value or the change in PNI (Additional file 12: Figure S10).

\section{Association between disease-free survival and $\triangle P N I$ based on tumor characteristics}

To investigate whether the effect of $\Delta$ PNI on diseasefree survival depends on tumor characteristics, we divided the patients according to ER and HER2 expression on tumors. In the ER-positive population, the high $\Delta \mathrm{PNI}$ group had significantly poorer disease-free survival than the low $\triangle$ PNI group $(p=0.030)$ (Fig. 4a). Meanwhile, as for HER2 status, the high $\triangle \mathrm{PNI}$ group presented significantly poorer disease-free survival than the low $\Delta \mathrm{PNI}$ group among HER2-negative cases $(p=0.029)$ (Fig. 4b). Disease-free survival was not significantly associated with ER negative $(p=0.32)$ and HER2 positive $(p=0.48)$ status, but the high $\triangle \mathrm{PNI}$ group tended to have poorer disease-free survival than the low $\triangle \mathrm{PNI}$ group in both the ER-negative and HER2-positive cohorts (Fig. 4a,b).

On division into four subtypes (luminal; ER+ and/or PgR+ / HER2-, luminal HER2: ER+ and/or PgR+ / HER2+, HER2 enriched: ER- and PgR- / HER2+, and TNBC: ER- and PgR-/HER-), the high $\triangle \mathrm{PNI}$ group showed a trend of poorer disease-free survival than the low $\triangle \mathrm{PNI}$ group, although the differences were not significant because of the small number of patients with each subtype ( $p=0.091$ for luminal, $p=0.098$ for luminal a

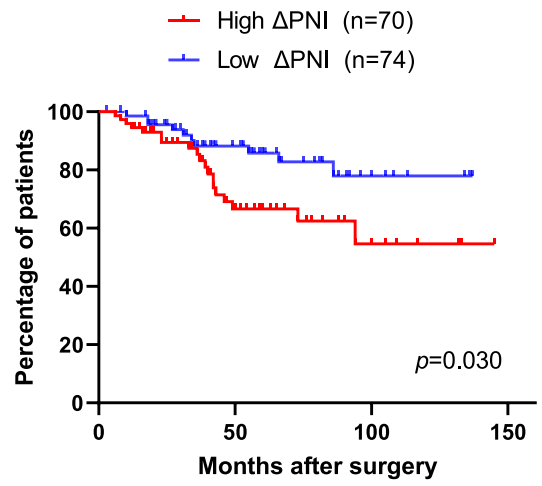

HR:2.27 (95\% Cl:1.09-4.71)

b

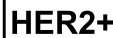

- High $\triangle \mathrm{PNI}(\mathrm{n}=30)$

- Low $\triangle \mathrm{PNI}(\mathrm{n}=31)$

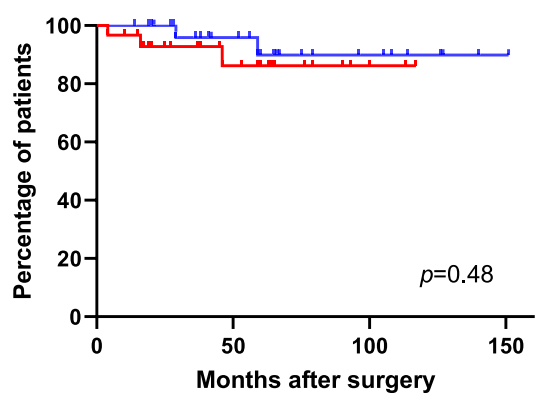

HR:1.86 (95\%Cl:0.32-10.90)
ER-

- High $\triangle \mathrm{PNI}(\mathrm{n}=21)$

- Low $\triangle \mathrm{PNI}(\mathrm{n}=26)$

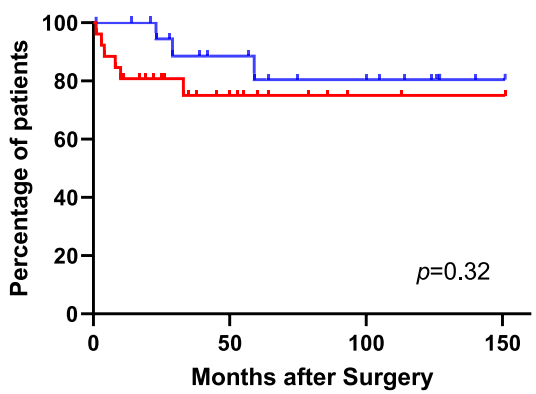

HR:1.96 (95\%Cl:0.53-7.26)

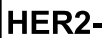

- High $\triangle \mathrm{PNI}(\mathrm{n}=70)$

- Low $\Delta \mathrm{PNI}(\mathrm{n}=60)$

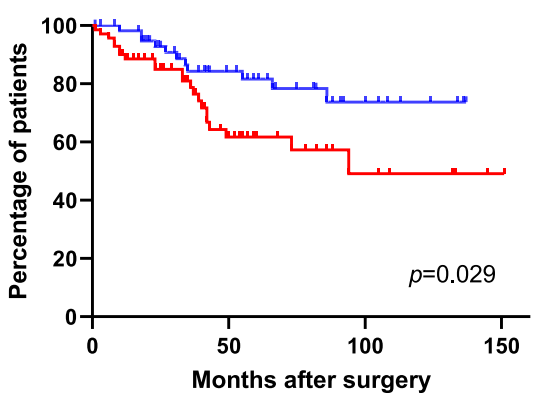

HR:2.17 (95\%Cl:1.10-4.31)

Fig. 4 Kaplan-Meier curves for DFS according to the change of PNI distributed by ER and HER2. a ER-positive $(p=0.030)$ and negative $(p=0.32)$ breast cancer. $\mathbf{b}$ HER2-positive $(p=0.48)$ and negative $(p=0.029)$ breast cancer. DFS: Disease-free survival, PNI: Prognostic nutritional index, ER: estrogen receptor, HER2: human epidermal growth factor receptor 2 
HER2, $p=0.67$ for HER2 enriched, and $p=0.18$ for TNBC) (Additional file 13: Figure S11).

Regarding clinical stage, the high $\triangle \mathrm{PNI}$ group showed significantly poorer disease-free survival than the low $\triangle \mathrm{PNI}$ group among patients with stage III breast cancer $(p=0.0064)$. In patients with stage I or II breast cancer, the high $\triangle \mathrm{PNI}$ group tended to show poorer disease-free survival than the low $\triangle \mathrm{PNI}$ group, although the difference was not significant $(p=0.39)$. As for HG, the high $\triangle \mathrm{PNI}$ group consistently showed poorer disease-free survival with respect to each HG with marginal or significant differences $(p=0.048$ for HG1, $p=0.072$ for HG2, $p=0.069$ for HG3) (Additional file 14: Figure S12).

\section{Prognostic factors of disease-free survival}

To confirm the significance of $\Delta \mathrm{PNI}$ in disease-free survival, univariate and multivariate analyses were performed. Univariate analysis revealed that $\triangle \mathrm{PNI}$ was a significant predictor of disease-free survival (HR: 2.2, 95\% CI: 1.14-4.41, $p=0.018$ ). Other factors associated with disease-free survival were pre-NAC clinical stage (HR: 3.1, 95\% CI: 1.58-5.81, $p<0.01$ ) and HER2 status (HR: 0.3, 95\% CI: 0.11-0.77, $p=0.012$ ). On multivariate analysis using Cox hazard model, $\triangle \mathrm{PNI}$ was an independent risk factor for disease-free survival (HR: 2.17, 95\% CI: 1.08-4.76, $p=0.042$ ) (Table 3).

\section{Discussion}

The present study demonstrates that high $\Delta \mathrm{PNI}$ is significantly associated with poor disease-free survival and is an independent predictor of disease-free survival. To the best of our knowledge, this is the first report to demonstrate that high $\triangle \mathrm{PNI}$ is a reliable prognostic factor of disease-free survival in patients with breast cancer who underwent NAC.
Several parameters, including PNI [3], serum albumin level [19], or NLR [20], are used to evaluate nutritional status. Increasing evidence suggests that high preoperative PNI is a predictor of better postoperative complications and patients outcomes in various types of malignancy [4-10]. Meanwhile, both high serum albumin level and low preoperative NLR also have been reported to be associated with better postoperative outcomes in several cancers [19-27]. BMI is also a wellknown prognostic factor in breast cancers [28-30], and body weight is also associated with the patients' nutritional condition [31]. These four factors (i.e., PNI, serum albumin level, NLR, and BMI) are easily calculated or obtained from clinical records or physical examinations. Therefore, we used these four factors as nutritional parameters in the present study.

We found no association between pre-NAC PNI, serum albumin level, NLR, or BMI and disease-free survival. Furthermore, post-NAC PNI, serum albumin level, NLR, or BMI also did not show any correlation with disease-free survival, although the low post-NAC PNI group tended to present poorer disease-free survival than the high post-NAC PNI group. These data indicated that the nutritional index itself did not predict the prognosis either before or after NAC. In general, chemotherapy worsen patients' nutritional condition due to its adverse gastrointestinal effects [15]. Although some studies have demonstrated significant decreases of various nutritional parameters such as albumin, pre-albumin, and transferrin due to preoperative chemotherapy in cancers of the digestive tract $[16,32]$, the influence of NAC on the nutritional status of patients with breast cancer has remained unclear. In the present study, we observed significant decreases in PNI and serum albumin level and increases in NLR after NAC. Particularly, PNI was

Table 3 Univariate and multivariate Cox proportional hazards regression analyses of the clinicopathological parameters

\begin{tabular}{|c|c|c|c|c|c|c|}
\hline & \multicolumn{3}{|c|}{ Univariate } & \multicolumn{3}{|c|}{ Multivariate } \\
\hline & $p$ value & $H R$ & $95 \% \mathrm{Cl}$ & $p$ value & $H R$ & $95 \% \mathrm{Cl}$ \\
\hline Age, years ( $\geq 50$ vs. $<50)$ & 0.73 & 0.89 & $0.47-1.68$ & & & \\
\hline Pre-NAC clinical stage (stage I and II vs. stage III) & $<0.01$ & 3.1 & $1.58-5.81$ & $<0.01$ & 2.17 & $1.57-7.27$ \\
\hline ER (positive vs. negative) & 0.97 & 1.1 & $0.48-2.14$ & & & \\
\hline HER2 (positive vs. negative) & 0.012 & 0.3 & $0.11-0.77$ & 0.016 & 0.28 & $0.10-0.79$ \\
\hline$\triangle \mathrm{PNI}$ (high vs. low) & 0.018 & 2.2 & $1.14-4.41$ & 0.042 & 2.17 & $1.08-4.76$ \\
\hline$\Delta$ Alb (high vs. low) & 0.058 & 1.9 & $0.97-3.52$ & & & \\
\hline$\triangle B M I$ (high vs. low) & 0.66 & 0.87 & $0.45-1.63$ & & & \\
\hline$\Delta N L R$ (high vs. low) & 0.66 & 1.2 & $0.61-2.18$ & & & \\
\hline Histological type (IDC vs. ILC or special type) & 0.23 & 2.4 & $0.58-10.03$ & & & \\
\hline Pathological response to NAC (non-pCR vs. pCR) & 0.19 & 0.64 & $0.32-1.25$ & & & \\
\hline
\end{tabular}

ER Estrogen receptor, HER-2 Human epidermal growth factor receptor type 2, BMI Body mass index, NLR Neutrophil/lymphocyte ratio, PNI Prognostic nutritional index, IDC Invasive ductal carcinoma, ILC Invasive lobular carcinoma, NAC Neoadjuvant chemotherapy, $p C R$ Pathological complete response 
decreased in 181 patients of 191 (94.7\%) after NAC. These results suggest that NAC has a negative effect on the nutritional status of patients with breast cancer, and that among the four commonly used parameters, PNI may be the most sensitive parameter to evaluate the nutritional status in patients with breast cancer. Therefore, we consequently focused on changes in the value of these nutritional parameters and found that a decreased PNI after NAC predicts poorer disease-free survival in patients with breast cancer. Significant differences in disease-specific survival were not observed in the present study. However, a trend of poorer disease-specific survival was observed in patients with a high decrease in PNI. A larger-scale study or longer follow-up periods will be able to reveal the differences in disease-specific survival.

In the comparison of clinicopathological characteristics between the high $\triangle \mathrm{PNI}$ group and the low $\Delta \mathrm{PNI}$ group, there was a higher percentage of patients who received DOC-containing regimens in the high $\triangle \mathrm{PNI}$ group than the low $\triangle \mathrm{PNI}$ group. However, there was no significant difference in disease-free survival among the three NAC regimens (AC only, AC followed by PTX and/or trastuzumab, and AC followed by DOC and/or trastuzumab), indicating that the significant difference in disease-free survival according to $\triangle \mathrm{PNI}$ does not depend on the type of chemotherapy regimen. One explanation for the higher number of patients who underwent DOC-containing regimens in the high $\triangle \mathrm{PNI}$ group may simply be due to the stronger gastrointestinal adverse effects of DOC compared with PTX [33, 34]. On the other hand, patients treated with DOC are likely to develop peripheral edema [33, 35], which is associated with hypoalbuminemia. This can be another explanation for the increase of DOC-treated patients in the high $\triangle \mathrm{PNI}$ group.

The biology of breast cancer is known to depend largely on its intrinsic subtype, which is determined mainly according to ER and HER2 status. Further, it is globally accepted that the prognosis is different between each subtype, and thus the therapeutic strategy depends on the subtype [36]. However, the nutritional status of patients with breast cancer may largely depend on patient factors, and not of the tumor. Consistent with this notion, the present study demonstrated that the influence of $\triangle$ PNI on disease-free survival may be similar across all breast cancer subtypes, particularly in patients with ER-positive or HER2-negative breast cancer; however, this should be interpreted cautiously as there was no statistical significance in the number of patients with different subtypes owing to the small number of patients enrolled in this study. Particularly, patients with HER2positive breast cancer had markedly good disease-free survival to evaluate the statistical difference between those with high and low $\Delta$ PNI. This may be due to the administration of trastuzumab that contributed to improved prognosis in patients with HER2-positive breast cancer [37]. As well as intrinsic subtype, clinical stage and HG are also universally accepted as prognostic factors of breast cancer $[38,39]$. This study showed that the influence of $\triangle \mathrm{PNI}$ on disease-free survival is stronger in the advanced stage, although the pre NAC-PNI, post NAC-PNI, and $\triangle \mathrm{PNI}$ values itself were not dependent on clinical stage. In addition, high $\triangle \mathrm{PNI}$ is consistently associated with poorer disease-free survival, independent from HG. Although further large-scale studies are required for determining the importance of nutritional change in patient outcomes according to the cancer subtype or the tumor burden, the results of the present study suggest that the association between changes in nutritional status during NAC and patient outcome mainly depends on the patient's nutritional status, especially in the advanced stage, but not on tumor characteristics.

From the point of view of immunity, better immunological condition has been considered to lead to improved survival in cancer. Malnutrition has been shown to be related to cancer progression due to its association with weak immune response [40, 41]. Accordingly, immune response has also been shown to correlate with better outcomes during various antitumor therapies in breast cancer [42]. Collectively, the result of the present and previous studies supports that maintaining the PNI during NAC may be beneficial to prevent worse prognosis in patients with breast cancer. Several studies have demonstrated that nutritional support such as supplemental immunonutrition containing $\mathrm{n}-3$ polyunsaturated fatty acids enabled improved the nutritional condition of patients who underwent chemotherapy [43-45]. Individual nutritional counseling has also been demonstrated to be important in maintaining the nutritional status [46]. In line with our findings, providing these nutritional support strategies during NAC may result in better patient outcome by maintaining the nutritional condition. Indeed, several clinical trials are ongoing to test whether nutrition interventions could improve the treatment outcome of metastatic breast cancer patients (NCT03045289, NCT03045289). In line with the results of this study, the concept of nutrition intervention should be further broadened to the neoadjuvant setting.

Several limitations of the present study need to be considered. First, it was a retrospective analysis with a small study population in a single institution. In addition to the heterogeneous nature of breast cancer, the limited number of patients may reduce the statistical power. Second, the NAC regimens varied between patients because the study period spanned several years when treatment regimens changed. Further investigations are therefore needed to validate our results. 


\section{Conclusions}

The findings of the present study indicate that a decrease of PNI can be a marker to predict poor prognosis after NAC in patients with breast cancer. Our results imply the importance of monitoring the nutritional status during NAC.

\section{Supplementary information}

Supplementary information accompanies this paper at https://doi.org/10. 1186/s12885-020-6647-4

Additional file 1: Figure S1. Box-and-whisker plot for Alb, NLR, and BMI in pre-NAC and post-NAC. NAC: Neoadjuvant chemotherapy, Alb: Serum albumin level ( $\mathrm{g} / \mathrm{dl}$ ), NLR: Neutrophil/lymphocyte ratio, BMI: Body mass index.

Additional file 2: Table S1. Distribution of patients with decreased PNI, Alb, and BMI or increased NLR during NAC.

Additional file 3: Table S2. The AUC and sensitivity/specificity for ROC curve.

Additional file 4: Figure S2. Disease-free survival evaluated using the Kaplan-Meier method for Alb, NLR, and BMI at pre-NAC. NAC: Neoadjuvant chemotherapy, Alb: Serum albumin level (g/dl), NLR: Neutrophil/ lymphocyte ratio, BMI: Body mass index.

Additional file 5: Figure $\mathbf{S 3}$. Disease-free survival evaluated using the Kaplan-Meier method for Alb, NLR, and BMI at post-NAC. NAC: Neoadjuvant chemotherapy, Alb: Serum albumin level (g/dl), NLR: Neutrophil/ lymphocyte ratio, BMI: Body mass index.

Additional file 6: Figure S4. Disease-specific survival evaluated using the Kaplan-Meier method for Alb, NLR, and BMI at pre-NAC and postNAC. NAC: Neoadjuvant chemotherapy, PNI: Prognostic nutritional index, Alb: Serum albumin level (g/dl), NLR: Neutrophil/lymphocyte ratio, BMl: Body mass index.

Additional file 7: Figure S5. Disease-specific survival evaluated using the Kaplan-Meier method according to change of PNI value. PNI: Prognostic nutritional index.

Additional file 8: Figure S6. Kaplan-Meier curves for disease-free survival according to change in Alb, NLR, and BMI. Alb: Serum albumin level ( $\mathrm{g} / \mathrm{dl})$, NLR: Neutrophil/lymphocyte ratio, BMI: Body mass index.

Additional file 9: Figure S7. Kaplan-Meier curves for disease-specific survival according to change in Alb, NLR, and BMI. Alb: Serum albumin level (g/dl), NLR: Neutrophil/lymphocyte ratio, BMI: Body mass index.

Additional file 10: Figure S8. Disease-free survival evaluated using the Kaplan-Meier method according to NAC regimens. NAC: Neoadjuvant chemotherapy, AC: Anthracycline, PTX: paclitaxel, DOC: Docetaxel.

Additional file 11: Figure S9. Box-and-whisker plot for $\triangle \mathrm{PNI}$ stratified by pre-NAC PNI. NAC: Neoadjuvant chemotherapy, PNI: Prognostic nutritional index.

Additional file 12: Figure S10. Box-and-whisker plot for pre-NAC PNI, post-NAC PNI, and $\triangle$ PNI stratified by clinical stage. NAC: Neoadjuvant chemotherapy, PNI: Prognostic nutritional index.

Additional file 13: Figure S11. Kaplan-Meier curves for disease-free survival according to change of PNI by breast cancer subtype. PNI: Prognostic nutritional index.

Additional file 14: Figure S12. Kaplan-Meier curves for disease-free survival according to change of PNI by clinical stage and HG. PNI: Prognostic nutritional index, HG: Histological grade.

\section{Abbreviations}

AC: Anthracycline; Alb: Serum albumin level; BMI: Body mass index; DOC: Docetaxel; EC: Epirubicin and cyclophosphamide; ER: Estrogen receptor; FEC: Fluorouracil, epirubicin, and cyclophosphamide; HER2: Human epidermal growth factor receptor type 2; HG: Histological grade; NAC: Neoadjuvant chemotherapy; NLR: Neutrophil/lymphocyte ratio; pCR: Pathological complete response; PgR: Progesterone receptor; PNI: Prognostic nutritional index; PTX: Paclitaxel; ROC: Receiver operating characteristics; TNBC: Triplenegative breast cancer

\section{Acknowledgements}

We would like to thank Editage (www.editage.com) for English language editing.

\section{Authors' contributions}

TO and KI designed the study. TO, DT, MO, TI, TK, KM collected the clinical data. TO performed the statistical analysis. The draft manuscript was prepared by TO and KM. All authors read and approved the final manuscript.

\section{Funding}

This work was not funded by any grant.

\section{Availability of data and materials}

The data supporting the findings of this work are available from the authors upon reasonable request.

\section{Ethics approval and consent to participate}

This study was approved by the Medical Ethics Committee on Clinical Investigation of Shinshu University (no. 4077). Patients were provided the opportunity to opt out of participation in this study via notifications displayed in the outpatient ward and the institution's website. Unless patients reject the enrollment in this study, the requirement for written informed consent was waived by the Medical Ethics Committee on Clinical Investigation of Shinshu University.

\section{Consent for publication}

Not applicable.

\section{Competing interests}

The authors declare that they have no competing interests.

Received: 10 July 2019 Accepted: 17 February 2020

Published online: 27 February 2020

References

1. Siegel R, Ma J, Zou Z, Jemal A. Cancer statistics, 2014. CA Cancer J Clin. 2014:64:9-29.

2. Rock CL, Doyle C, Demark-Wahnefried W, Meyerhardt J, Courneya KS, Schwartz $A L$, et al. Nutrition and physical activity guidelines for cance survivors. CA Cancer J Clin. 2012;62:243-74.

3. Onodera T, Goseki N, Kosaki G. Prognostic nutritional index in gastrointestinal surgery of malnourished cancer patients. Nihon Geka Gakkai Zasshi. 1984:85:1001-5.

4. Yang Y, Gao P, Song Y, Sun J, Chen X, Zhao J, et al. The prognostic nutritional index is a predictive indicator of prognosis and postoperative complications in gastric cancer: a meta-analysis. Eur J Surg Oncol. 2016;42: 1176-82.

5. Yang $Y$, Gao P, Chen X, Song Y, Shi J, Zhao J, et al. Prognostic significance of preoperative prognostic nutritional index in colorectal cancer: results from a retrospective cohort study and a meta-analysis. Oncotarget. 2016;7: 58543-52.

6. Shoji F, Morodomi Y, Akamine T, Takamori S, Katsura M, Takada K, et al. Predictive impact for postoperative recurrence using the preoperative prognostic nutritional index in pathological stage I non-small cell lung cancer. Lung Cancer. 2016:98:15-21.

7. Qiu C, Qu X, Shen H, Zheng C, Zhu L, Meng L, et al. Evaluation of prognostic nutritional index in patients undergoing radical surgery with nonsmall cell lung Cancer. Nutr Cancer. 2015;67:741-7.

8. Mori S, Usami N, Fukumoto K, Mizuno T, Kuroda H, Sakakura N, et al. The significance of the prognostic nutritional index in patients with completely resected non-small cell lung Cancer. PLoS One. 2015;10:e0136897.

9. Kanda M, Fujii T, Kodera Y, Nagai S, Takeda S, Nakao A. Nutritional predictors of postoperative outcome in pancreatic cancer. Br J Surg. 2011;98:268-74.

10. Broggi MS, Patil D, Baum Y, Nieh PT, Alemozaffar M, Pattaras JG, et al. Onodera's prognostic nutritional index as an independent prognostic factor in clear cell renal cell carcinoma. Urology. 2016;96:99-105. 
11. Mohri T, Mohri Y, Shigemori T, Takeuchi K, Itoh Y, Kato T. Impact of prognostic nutritional index on long-term outcomes in patients with breast cancer. World J Surg Oncol. 2016;14:170.

12. Yang Z, Zhang B, Hou L, Xie $Y$, Cao X. Pre-operative prognostic nutritional index predicts the outcomes for triple-negative breast cancer. Tumour Biol. 2014;35:12165-71.

13. Wolmark N, Wang J, Mamounas E, Bryant J, Fisher B. Preoperative chemotherapy in patients with operable breast cancer: nine-year results from National Surgical Adjuvant Breast and Bowel Project B-18. J Natl Cancer Inst Monogr. 2001;30:96-102.

14. von Minckwitz G, Untch M, Blohmer JU, Costa SD, Eidtmann H, Fasching PA, et al. Definition and impact of pathologic complete response on prognosis after neoadjuvant chemotherapy in various intrinsic breast cancer subtypes. J Clin Oncol. 2012;30:1796-804

15. Hiura Y, Takiguchi S, Yamamoto K, Takahashi T, Kurokawa Y, Yamasaki M, et al. Effects of ghrelin administration during chemotherapy with advanced esophageal cancer patients: a prospective, randomized, placebo-controlled phase 2 study. Cancer. 2012;118:4785-94.

16. Migita K, Matsumoto S, Wakatsuki K, Ito M, Kunishige T, Nakade H, et al. A decrease in the prognostic nutritional index is associated with a worse long-term outcome in gastric cancer patients undergoing neoadjuvant chemotherapy. Surg Today. 2017;47:1018-26.

17. Tomita M, Shimizu T, Ayabe T, Yonei A, Onitsuka T. Preoperative neutrophi to lymphocyte ratio as a prognostic predictor after curative resection for non-small cell lung cancer. Anticancer Res. 2011;31:2995-8.

18. Shimizu K, Okita R, Saisho S, Maeda A, Nojima Y, Nakata M. Preoperative neutrophil/lymphocyte ratio and prognostic nutritional index predict survival in patients with non-small cell lung cancer. World J Surg Oncol. 2015;13:291

19. Miura K, Hamanaka K, Koizumi T, Kitaguchi Y, Terada Y, Nakamura D, et al. Clinical significance of preoperative serum albumin level for prognosis in surgically resected patients with non-small cell lung cancer: comparative study of normal lung, emphysema, and pulmonary fibrosis. Lung Cancer. 2017;111:88-95.

20. Ethier JL, Desautels D, Templeton A, Shah PS, Amir E. Prognostic role of neutrophil-to-lymphocyte ratio in breast cancer: a systematic review and meta-analysis. Breast Cancer Res. 2017;19:2.

21. Templeton AJ, McNamara MG, Seruga B, Vera-Badillo FE, Aneja P, Ocana A, et al. Prognostic role of neutrophil-to-lymphocyte ratio in solid tumors: a systematic review and meta-analysis. J Natl Cancer Inst. 2014;106:dju124.

22. Song $Y$, Yang $Y$, Gao $P$, Chen $X, Y u$ D, Xu Y, et al. The preoperative neutrophil to lymphocyte ratio is a superior indicator of prognosis compared with other inflammatory biomarkers in resectable colorecta cancer. BMC Cancer. 2017;17:744.

23. Sharaiha RZ, Halazun KJ, Mirza F, Port JL, Lee PC, Neugut Al, et al. Elevated preoperative neutrophil:lymphocyte ratio as a predictor of postoperative disease recurrence in esophageal cancer. Ann Surg Oncol. 2011;18:3362-9.

24. Marin Hernandez C, Pinero Madrona A, Gil Vazquez PJ, Galindo Fernandez PJ, Ruiz Merino G, Alonso Romero JL, et al. Usefulness of lymphocyte-tomonocyte, neutrophil-to-monocyte and neutrophil-to-lymphocyte ratios as prognostic markers in breast cancer patients treated with neoadjuvant chemotherapy. Clin Transl Oncol. 2018;20:476-83.

25. Ma J, Kuzman J, Ray A, Lawson BO, Khong B, Xuan S, et al. Neutrophil-tolymphocyte ratio (NLR) as a predictor for recurrence in patients with stage III melanoma. Sci Rep. 2018;8:4044.

26. Iwase T, Sangai T, Sakakibara M, Sakakibara J, Ishigami E, Hayama S, et al. An increased neutrophil-to-lymphocyte ratio predicts poorer survival following recurrence for patients with breast cancer. Mol Clin Oncol. 2017;6:266-70.

27. Azab B, Bhatt VR, Phookan J, Murukutla S, Kohn N, Terjanian T, et al. Usefulness of the neutrophil-to-lymphocyte ratio in predicting short- and long-term mortality in breast cancer patients. Ann Surg Oncol. 2012;19:217-24.

28. Ryu SY, Kim CB, Nam CM, Park JK, Kim KS, Park J, et al. Is body mass index the prognostic factor in breast cancer?: a meta-analysis. J Korean Med Sci. 2001;16:610-4

29. Protani M, Coory M, Martin JH. Effect of obesity on survival of women with breast cancer: systematic review and meta-analysis. Breast Cancer Res Treat. 2010;123:627-35

30. Chlebowski RT, Aiello E, McTiernan A. Weight loss in breast cancer patient management. J Clin Oncol. 2002;20:1128-43.

31. Chlebowski RT. Nutrition and physical activity influence on breast cance incidence and outcome. Breast. 2013;22(Suppl 2):S30-7.
32. Yoshida N, Watanabe M, Baba Y, Ishimoto T, Iwagami S, Sakamoto Y, et al. Influence of preoperative docetaxel, cisplatin, and 5-fluorouracil on the incidence of complications after esophagectomy for resectable advanced esophageal cancer. Dis Esophagus. 2014:27:374-9.

33. Jones SE, Erban J, Overmoyer B, Budd GT, Hutchins L, Lower E, et al. Randomized phase III study of docetaxel compared with paclitaxel in metastatic breast cancer. J Clin Oncol. 2005;23:5542-51.

34. Sparano JA, Wang M, Martino S, Jones V, Perez EA, Saphner T, et al. Weekly paclitaxel in the adjuvant treatment of breast cancer. N Engl J Med. 2008; 358:1663-71.

35. Harvey V, Mouridsen H, Semiglazov V, Jakobsen E, Voznyi E, Robinson BA, et al. Phase III trial comparing three doses of docetaxel for second-line treatment of advanced breast cancer. J Clin Oncol. 2006:24:4963-70.

36. Coates AS, Winer EP, Goldhirsch A, Gelber RD, Gnant M, Piccart-Gebhart M, et al. Tailoring therapies-improving the management of early breast cancer: St Gallen international expert consensus on the primary therapy of early breast Cancer 2015. Ann Oncol. 2015;26:1533-46.

37. Gianni L, Eiermann W, Semiglazov V, Manikhas A, Lluch A, Tjulandin S, et al. Neoadjuvant chemotherapy with trastuzumab followed by adjuvant trastuzumab versus neoadjuvant chemotherapy alone, in patients with HER2-positive locally advanced breast cancer (the NOAH trial): a randomised controlled superiority trial with a parallel HER2-negative cohort. Lancet. 2010;375:377-84.

38. DeSantis CE, Ma J, Goding Sauer A, Newman LA, Jemal A. Breast cancer statistics, 2017, racial disparity in mortality by state. CA Cancer J Clin. 2017; 67:439-48.

39. Rakha EA, Reis-Filho JS, Baehner F, Dabbs DJ, Decker T, Eusebi V, et al. Breast cancer prognostic classification in the molecular era: the role of histological grade. Breast Cancer Res. 2010;12:207.

40. Zitvogel L, Pietrocola F, Kroemer G. Nutrition, inflammation and cancer. Nat Immunol. 2017:18:843-50.

41. Gupta D, Lis CG. Pretreatment serum albumin as a predictor of cancer survival: a systematic review of the epidemiological literature. Nutr J. 2010;9: 69.

42. Mahmoud SM, Paish EC, Powe DG, Macmillan RD, Grainge MJ, Lee AH, et al. Tumor-infiltrating CD8+ lymphocytes predict clinical outcome in breast cancer. J Clin Oncol. 2011:29:1949-55.

43. Murphy RA, Mourtzakis M, Chu QS, Baracos VE, Reiman T, Mazurak VC. Supplementation with fish oil increases first-line chemotherapy efficacy in patients with advanced nonsmall cell lung cancer. Cancer. 2011;117:377480.

44. Xue H, Sawyer MB, Field CJ, Dieleman LA, Baracos VE. Nutritional modulation of antitumor efficacy and diarrhea toxicity related to irinotecan chemotherapy in rats bearing the ward colon tumor. Clin Cancer Res. 2007; 13:7146-54

45. Kiss NK, Krishnasamy M, Isenring EA. The effect of nutrition intervention in lung cancer patients undergoing chemotherapy and/or radiotherapy: a systematic review. Nutr Cancer. 2014;66:47-56.

46. Ravasco P, Monteiro-Grillo I, Camilo M. Individualized nutrition intervention is of major benefit to colorectal cancer patients: long-term follow-up of a randomized controlled trial of nutritional therapy. Am J Clin Nutr. 2012;96: 1346-53.

\section{Publisher's Note}

Springer Nature remains neutral with regard to jurisdictional claims in published maps and institutional affiliations.

Ready to submit your research? Choose BMC and benefit from:

- fast, convenient online submission

- thorough peer review by experienced researchers in your field

- rapid publication on acceptance

- support for research data, including large and complex data types

- gold Open Access which fosters wider collaboration and increased citations

- maximum visibility for your research: over $100 \mathrm{M}$ website views per year

At $\mathrm{BMC}$, research is always in progress.

Learn more biomedcentral.com/submissions 